\title{
HÁBITOS DE CONSUMO E AGENDA DE INOVAÇÃO: CONTRIBUIÇÕES PARA A INVESTIGAÇÃO QUALITATIVA POR MEIO DA APLICAÇÃO DE UM MARCO PRELIMINAR A UM CASO DE ESTUDO
}

\author{
Dioclecio Cameloㄹ, Carolina Daros ${ }^{2}$ e André de Souza Lucca ${ }^{3}$ \\ 1Universidade Estadual de Maringá, Brasil. dino@dioclecio.com \\ ${ }^{2 e} 3$ Universidade Tecnológica Federal do Paraná, Brasil. carolinadaros@gmail.com, alucca@utfpr.edu.br
}

\begin{abstract}
Resumo. Conhecer os hábitos de consumo de um determinado público é fundamental para orientar qualquer desenvolvimento de novo produto no design. Propor mudanças nesses hábitos orientando-os para a inovação é um desafio para novos projetos. Este estudo apresenta a experiência adquirida com a aplicação de um marco preliminar de pesquisa orientado para designers. Por meio desta experiência foi possível identificar os hábitos de consumo de famílias de baixa renda, residentes em Habitações de Interesse Social. Esse levantamento ajudou a estabelecer uma proposta de agenda de inovação apoiada em mudanças nos hábitos de consumo de água e energia visando a otimização das atividades domésticas. Essa experiência permitiu conhecer a importância dos métodos de pesquisa, sobretudo da etnografia, como ferramenta de apoio ao desenvolvimento de novos produtos e de políticas públicas orientadas a promover um consumo sustentável. Após a aplicação, foram identificadas melhorias no marco e a possibilidade incluir novos instrumentos para evoluir o marco proposto em um modelo teórico de pesquisa pautado nos métodos mistos. Com esse aprimoramento, espera-se orientar os designers na elaboração de requisitos amplos, porém próximos das demandas dos consumidores, direcionando o desenvolvimento de soluções na indústria e em ações do poder público.
\end{abstract}

Palavras-chave: Pesquisa Qualitativa; Design de Produtos; Políticas Públicas; Métodos de Pesquisa; Hábitos de Consumo.

\section{IDENTIFICATION OF HABITS OF CONSUMPTION: APPLICATION OF A FRAMEWORK TO A CASE STUDY}

Abstract. Knowing the consumption habits of a certain public is fundamental to lead any design development. Proposing changes to achieve these habits is a challenge for new design projects. This study reports the experience learned with the application of a preliminary research framework oriented towards designers. From this study it was possible to identify the consumption habits of low-income families living in Social Housing. This research assisted in establishing a proposal for an innovation agenda to promote changes in consumption habits regarding the optimized use of water and energy in domestic activities. This experience allowed us to learn about the importance of research methods, especially ethnographic instruments, as tools to support the development of new products and public policies. After the results were applied, improvements were identified in the framework and the possibility of including new instruments to evolve in a theoretical research model based on qualitative methods and ethnography. With this refinement, it is expected to guide designers in the elaboration of broader requirements, but closer to consumer demands, directing the development of solutions in industry and in actions of public administration.

Keywords: Design Research; Product Design; Public Policy; Research Methods; Habits of Consumption. 


\section{INTRODUÇÃO}

O processo de desenvolvimento de produtos com uma proposta sustentável pode considerar inicialmente a compreensão dos hábitos de consumo de seus usuários dentro de seu contexto e cotidiano.

Estima-se que seja possível conhecer esses hábitos a partir da aplicação de determinadas estratégias, buscando com isso propor alternativas para que os consumidores consigam mudar hábitos e comportamentos para ações sustentáveis (Bocken, Mugge, Bom, \& Lemstra, 2018; Lilley, 2009).

Alguns estudos combinaram instrumentos de pesquisa para coletar, tratar e aplicar estratégias de análise para aprofundar o entendimento sobre os hábitos de consumo de determinado grupo de pessoas, localidades ou comunidades (Bocken et al., 2018; Chu, Glad, \& Wever, 2019; Chu, Steenstra, Wever, \& Glad, 2018).

Entretanto, raros são os estudos que propõem diretrizes projetuais, amplas e de longo prazo, com orientações para os atores envolvidos no desenvolvimento de novos produtos e políticas públicas.

A presente pesquisa foi orientada pela seguinte questão: quais são os recursos necessários para promover mudanças nos hábitos de consumo de água e energia a partir de contribuições feitas sob a ótica do design? Tomou-se como pressuposto que as pessoas realizam suas atividades domésticas e de higiene de forma automática. Tal pressuposto também considera que essas atividades são mediadas por produtos e serviços que apresentam limitações relacionadas ao consumo de água e energia.

Outro pressuposto desse estudo é que existem diretrizes metaprojetuais para implementar os princípios do design para a sustentabilidade orientada a otimização do consumo de água e energia nas Habitações de Interesse Social (HIS), assim como o estabelecimento de diretrizes metaprojetuais que foram os objetivos da presente pesquisa.

Nesse contexto, os autores oferecem um marco preliminar para o desenvolvimento de pesquisas que envolvem a compreensão dos hábitos de consumo até a proposição de uma agenda de inovação. 
Essa agenda deverá corresponder no agrupamento de diretrizes metaprojetuais que orientem o desenvolvimento de produtos e a elaboração de políticas públicas buscando modificar hábitos de consumo.

O público-alvo da agenda de inovação são os designers, a indústria e gestores do poder público. Como forma de conhecer sua efetividade, este marco foi aplicado na Comunidade Mirante do Iguaçu, no município de Balsa Nova, Região metropolitana de Curitiba, Paraná, Brasil (Daros, 2013).

Por meio dessa aplicação, buscou-se compreender os hábitos de consumo de água e energia de famílias de baixa renda residentes em Habitações de Interesse Social em suas atividades domésticas e de higiene.

Para atender esses aspectos, o presente artigo descreve o marco preliminar de forma breve. Em seguida, apresenta o resultado obtido na sua aplicação.

Depois, as discussões sobre o aprendizado são realizadas indicando sugestões de aprimoramento da proposta de marco preliminar. Por fim, algumas conclusões são traçadas destacando as contribuições do estudo de caso e sugerindo alguns trabalhos futuros.

\subsection{Marco preliminar de pesquisa}

O marco preliminar foi composto de duas partes. A primeira destaca as ações ( 1 a 4 ) destinadas a explorar e compreender os hábitos de consumo e o contexto em que o consumidor utiliza os recursos. E a segunda ( 5 a 7 ) visa dar suporte ao desenvolvimento de diretrizes metaprojetuais baseadas em cenários futuros sustentáveis.

O Quadro 1 indica uma síntese das atividades consideradas na proposta deste marco.

O marco preliminar foi composto por 7 atividades, cada qual com o seu objetivo, indicação de instrumentos para coleta de dados, tratamento e estratégia para análise dos dados.

Após a estruturação do marco preliminar, o mesmo foi aplicado em um estudo de caso, conforme descrição dos tópicos a seguir. 


\section{MATERIAIS E MÉTODOS}

O marco preliminar foi proposto para atender ao Projeto E-Wise, desenvolvido pelo Núcleo de Design e Sustentabilidade (NDS), da Universidade Federal do Paraná (UFPR), como parte do projeto Rede 22 - Uso racional de água e eficiência energética em habitação de interesse social, aprovado na Chamada Pública Saneamento Ambiental e Habitação 07/2009, e recebeu financiamento da FINEP (Financiadora de Estudos e Projetos) (Daros, 2013; Daros, Camelo, Silvério, \& Santos, 2014). A elaboração do marco preliminar iniciou com uma revisão da literatura destacando aspectos sobre o design sustentável onde foram especificadas as características que podem ser indicadas em produtos e serviços (Lilley, 2007; Tang \& Bhamra, 2008; Vezzoli \& Manzini, 2008), aspectos que conduzem a inovação (Celaschi \& Deserti, 2007; Marinova, 2009; Tamborrini, 2014), prospecção de cenários e produtos (Godet, 2001; Porter et al., 2004). Além disso, foram analisados trabalhos que exploram e aprofundam os hábitos de consumo, como Chu $(2019,2018)$ que trata sobre a retenção do comportamento sustentável, Bocken (2018) que estuda sobre o impacto do sistema pay-per-use no comportamento, Lockton $(2010,2016)$ que trata sobre a influência de cartões inspiracionais, Elizondo (2011) que estuda as influências culturais nos hábitos de consumo, Barr (2011) que aborda sobre a influência do marketing social sobre o consumo e Lucero (2007), que estudou o consumo da iluminação doméstica. Nessa análise, foram identificados e comparados os instrumentos e procedimentos adotados na coleta e tratamento dos dados dos consumidores, além disso foram identificadas as estratégias consideradas na interpretação dos comportamentos de consumo de determinado público. Após a elaboração, o marco preliminar foi aplicado em uma comunidade de baixa renda. $A$ equipe composta para a execução desta pesquisa foi formada pelo coordenador geral (PhD.), um pesquisador (PhD.), uma mestranda, um aluno de iniciação científica. $\mathrm{E}$ os resultados dessa aplicação foram analisados para indicar melhorias nos instrumentos estabelecidos e nas formas para obter informações relevantes para a pesquisa.

\section{RESULTADOS}

Os próximos tópicos abordam os resultados obtidos na aplicação do marco referencial de pesquisa, assim como apontam as melhorias. Parte expressiva da presente seção mostra uma análise da aplicação do marco preliminar a partir das práticas registradas nos trabalhos de Daros e Santos (2013; 2014). 
Quadro 1: Resumo do marco preliminar. Fonte: (Os autores).

\begin{tabular}{|c|c|c|c|}
\hline $\begin{array}{l}\text { Etapa/Nome da } \\
\text { etapa }\end{array}$ & Objetivo & Coleta de dados & Tratamento de dados e estratégia de análise (passo a passo) \\
\hline $\begin{array}{l}\text { 1. Definição do } \\
\text { público }\end{array}$ & $\begin{array}{l}\text { Definir o perfil do grupo a ser } \\
\text { pesquisado. }\end{array}$ & $\begin{array}{l}\text { Elaborar os critérios de seleção } \\
\text { da amostra com base em } \\
\text { requisitos geográficos e } \\
\text { demográficos. Levantar as } \\
\text { comunidades compativeis. }\end{array}$ & $\begin{array}{l}\text { 1. Averiguar se a comunidade é compativel com os critérios } \\
\text { pré-estabelecidos: localização geográfica, tipo e } \\
\text { características da residência, tipo de moradia, renda familiar, } \\
\text { número de residentes, famílias com ou sem crianças, pessoa } \\
\text { responsável pelas atividades domésticas. }\end{array}$ \\
\hline $\begin{array}{l}\text { 2. Exploração de } \\
\text { contextos }\end{array}$ & $\begin{array}{l}\text { Traçar um panorama geral sobre o } \\
\text { perfil do entrevistado e o contexto } \\
\text { que está inserido. }\end{array}$ & $\begin{array}{l}\text { Survey: elaborar o questionário } \\
\text { com a partcipação de } \\
\text { especialistas, sugere-se o método } \\
\text { Delphi. A estrutura do } \\
\text { questionário proposto: perfil do } \\
\text { entrevistado e da família, } \\
\text { características da habitação, } \\
\text { consumo de água e energia, } \\
\text { interesses (opiniões, } \\
\text { necessidades, desejos atuais e } \\
\text { futuros). }\end{array}$ & $\begin{array}{l}\text { 1. Tabulação em planilha eletrônica por frequência, } \\
\text { percentuais, médias de respostas e análise descritiva. } 2 \text {. } \\
\text { Análise AIO (Atividades, Interesses e Opiniões) (Vyncke, } \\
\text { 2002): cruzar os dados com o objetivo de identificar os perfis } \\
\text { dos entrevistados a partir do estilo de vida. 3. Definir e } \\
\text { selecionar pequenos grupos para o aprofundamento da } \\
\text { compreensão dos hábitos de consumo. } 4 \text {. Definir a linguagem } \\
\text { textual e visual a ser adotada nos instrumentos de coleta de } \\
\text { dados e sob quais condições poderão ser realizados. }\end{array}$ \\
\hline $\begin{array}{l}\text { 3. Pesquisa } \\
\text { etnográfica }\end{array}$ & $\begin{array}{l}\text { Compreensão aprofundada dos } \\
\text { hábitos, costumes, usos do objeto } \\
\text { investigado por um determinado } \\
\text { público ou comunidade. }\end{array}$ & $\begin{array}{l}\text { Sondas culturais: } 0 \\
\text { desenvolvimento do kit atendeu } \\
\text { a abordagem Attrakdiff (Ramos, } \\
\text { 2002). O kit das sondas culturais } \\
\text { foi composto por agenda de } \\
\text { tarefas e adesivos, câmera } \\
\text { fotográfica descartável, cartões } \\
\text { de avaliação de atividades }\end{array}$ & $\begin{array}{l}\text { 1. Análise dos dados em workshops com equipe } \\
\text { multidisciplinar, de preferência que tenham alguma relação } \\
\text { com a comunidade observada ou com as áreas de pesquisa } \\
\text { da investigação. 2. Agenda de tarefas e adesivos: tabulação } \\
\text { em planilha eletrônica e análise de frequência de cada } \\
\text { atividade doméstica e de higiene, e média semanal. } 3 \text {. } \\
\text { Fotografias: sequenciar as atividades em etapas. Observa-las } \\
\text { a partir de critérios previamente estabelecidos. } 4 \text {. Avaliação } \\
\text { de atividades: tabulação em planilha eletrônica, locação das } \\
\text { reações (sentimentos) do usuário na etapa da atividade } \\
\text { desenvolvida. }\end{array}$ \\
\hline $\begin{array}{l}4 . \\
\text { Entrevista }\end{array}$ & $\begin{array}{l}\text { Explorar as questões dos hábitos a } \\
\text { partir de perguntas do tipo como, } \\
\text { por que, qual a frequência, critérios } \\
\text { de compra e descarte. }\end{array}$ & $\begin{array}{l}\text { Entrevista semiestruturada: } \\
\text { aspectos da moradia, estilo de } \\
\text { vida, consumo dos } \\
\text { eletrodomésticos em relação a } \\
\text { água e energia, limpeza e higiene, } \\
\text { lavar roupa, passar roupa, lavar } \\
\text { louça, aspectos de higiene }\end{array}$ & $\begin{array}{l}\text { 1. Transcrição das informações relevantes, assim como as } \\
\text { situações enfatizadas, recorrência e pertinência aos hábitos } \\
\text { de consumo. } 2 \text {. Sistematizar os dados, buscando agrupá-los } \\
\text { por padrões de recorrência. 3. Elaboração de infográficos, } \\
\text { storyboard, jornada do usuário, blueprint em relação a cada } \\
\text { atividade. Sugere-se que a etapa de análise dos dados seja } \\
\text { feita por equipes multidisciplinares }\end{array}$ \\
\hline $\begin{array}{l}\text { 5. Banco de inovação } \\
\text { (roadmap) }\end{array}$ & $\begin{array}{l}\text { Oferecer um banco de imagens e } \\
\text { informações orientado a consulta } \\
\text { rápida de soluções já propostas e } \\
\text { que permite explorar características } \\
\text { específicas dos artefatos } \\
\text { investigados. }\end{array}$ & $\begin{array}{l}\text { Banco de inovação: foi utilizada a } \\
\text { plataforma Tiddlywiki (Ruston, } \\
\text { 2015) permitindo alimentar e } \\
\text { compartilhar informações em } \\
\text { rede privada usando navegadores } \\
\text { web. }\end{array}$ & $\begin{array}{l}\text { 1. Identificar e selecionar imagens e informações sobre } \\
\text { eletrodomésticos. } 2 \text {. Classificar e organizar do banco de } \\
\text { dados de inovações a partir de critérios, como por exemplo: } \\
\text { recursos utilizados, materiais, tecnologias. } 3 \text {. Visualizar e } \\
\text { analisar a evolução tecnológica, de forma, funcionalidades } \\
\text { dos produtos (eletrodomésticos), entre outras questões que } \\
\text { impactam as atividades domésticas. }\end{array}$ \\
\hline 6. Cenários futuros & $\begin{array}{l}\text { Idealizar e propor situações para um } \\
\text { contexto especifico. A partir disso, } \\
\text { identificar soluções para produtos e }\end{array}$ & $\begin{array}{l}\text { Cenários futuros: workshops para } \\
\text { a construção de cenários futuros } \\
\text { por meio da colaboração de } \\
\text { especialistas de diversas áreas do } \\
\text { conhecimento (tecnologias, água, } \\
\text { energia e sustentabilidade). } \\
\text { Idealização a partir de três } \\
\text { hipóteses de cenários: cenário de } \\
\text { crise, cenário mediano e cenário } \\
\text { sustentável para as atividades } \\
\text { domésticas e de higiene. }\end{array}$ & $\begin{array}{l}\text { 1. Descrição textual dos cenários idealizados. 2. Organizar e } \\
\text { agrupar as soluções para produtos e serviços para a atividade } \\
\text { de domésticas e de higiene por meio da "recorrência" e } \\
\text { "semelhança" de proposições. 3. Identificar e interpretar as } \\
\text { oportunidades de inovação representadas pelas soluções, } \\
\text { opiniões e ideias dos participantes do workshop de cenários } \\
\text { futuros. } 4 \text {. Classificar as ideias a partir de critérios e/ou } \\
\text { estratégias previamente estabelecidas. No caso da presente } \\
\text { pesquisa, tem-se critérios para água e energia (economia, } \\
\text { eficiência, suficiência, substituição, reaproveitamento), } \\
\text { hábitos de consumo (orientar a mudança, manter a mudança, } \\
\text { assegurar a mudança), sustentabilidade (redesign, novos } \\
\text { produtos sustentáveis, sistema produto-serviço, estilo de vida } \\
\text { sustentável), inovação (incremental, radical, mudança } \\
\text { tecnológica, revolução tecnológica, inovação de significado, } \\
\text { inovação social). }\end{array}$ \\
\hline $\begin{array}{l}\text { 7. Elaboração de } \\
\text { diretrizes } \\
\text { metaprojetuais }\end{array}$ & $\begin{array}{l}\text { Elaborar diretrizes orientadas a } \\
\text { sustentabilidade que atendem tanto } \\
\text { gestores públicos, para a criação de } \\
\text { políticas públicas, como designers e } \\
\text { indústrias, a partir de diretrizes } \\
\text { metaprojetuais, a serem } \\
\text { consideradas no processo de } \\
\text { desenvolvimento de novos } \\
\text { produtos, ou ao setor de serviços, } \\
\text { visando a mudança dos hábitos de } \\
\text { consumo. }\end{array}$ & $\begin{array}{l}\text { Utilizar os resultados do banco de } \\
\text { inovação e/ou cenários futuros } \\
\text { para elaboração das diretrizes } \\
\text { metaprojetuais }\end{array}$ & $\begin{array}{l}\text { 1. Elaborar as diretrizes a partir dos resultados do banco de } \\
\text { inovação e/ou cenários futuros. 2. Agrupar as diretrizes } \\
\text { metaprojetuais por categorias: artefatos, serviços, sistema } \\
\text { produto-serviço. E subcategorias: materiais, tecnologias, } \\
\text { dentre outros. 3. Organizar as diretrizes em uma agenda de } \\
\text { inovação de acordo com critérios sociais, ambientais, } \\
\text { tecnológicos, por exemplo. }\end{array}$ \\
\hline
\end{tabular}




\subsection{Aplicação do marco preliminar}

A aplicação do marco preliminar iniciou com uma delimitação do universo pesquisado. Essa etapa seguiu a orientação dos técnicos da Companhia de Habitação do Paraná (COHAPAR) que indicaram quais seriam as comunidades que atendiam o perfil explorado pela pesquisa. Com esse contato, optou-se por estudar a comunidade Mirante do Iguaçú, por estarem instalados a mais de 6 meses. Essa comunidade foi formada para atender especificamente a população de baixa renda e possui 81 HIS com $32 \mathrm{~m}^{2}$ voltadas à população de baixa renda.

A coleta dos dados iniciou com a aplicação de uma survey onde as famílias foram previamente contactadas e a proposta de estudo apresentada. Todos os participantes assinaram um termo de consentimento aceitando participar das atividades indicadas pela pesquisa. Das residências existentes, somente 60 famílias foram abordadas e 32 atendiam os requisitos de baixa renda indicados para o mínimo de três residentes por moradia. $A$ análise da survey traçou um panorama geral sobre os entrevistados, sobre as condições das habitações, o consumo de água e energia, e os seus interesses. Nessa pesquisa, predominaram as famílias onde as mulheres eram responsáveis pelos afazeres domésticos (97\%), o grau de escolaridade fundamental incompleto era predominante (54\%) e a renda mensal girava entre $R \$ 400$ a $R \$ 800$ (34\%), valores entre 125,00 e 250,00 euros na época. Verificou-se que as características das habitações, assim como as fontes de abastecimento de água e fornecimento de energia determinam e influenciam diretamente o consumo de água e energia nas residências. Na pesquisa também foram identificados os artefatos que estiveram presentes nas HIS, aqueles que consomem água e energia, as medidas de economia tomadas, as percepções sobre o consumo de água e energia, a baixa adesão a tarifa social e as despesas com esses recursos. Mesmo com os dados coletados houve necessidades de explorar particularidades sobre os hábitos e rotinas dentro das residências (Daros, 2013; Daros et al., 2014).

As sondas culturais foram aplicadas em 9 famílias selecionadas a partir de similaridades identificadas na frequência dos banhos e na higienização (lavagem e passagem) das roupas (figura 1). Com os dados das sondas analisados, constatou-se que os hábitos de consumo eram semelhantes em contextos análogos pois tinham como principal característica a rede pública para o fornecimento de água e energia. Como forma de economia, constatou-se que muitas famílias reaproveitavam a água dos tanques, tanquinhos ou lavadores para outros 
fins, dentro e fora das residências. Os dados coletados foram agrupados e contrastados com a análise das surveys. Em relação ao fornecimento de energia elétrica, não foram identificados meios alternativos de fornecimento ou geração de energia para alimentar os eletrodomésticos.
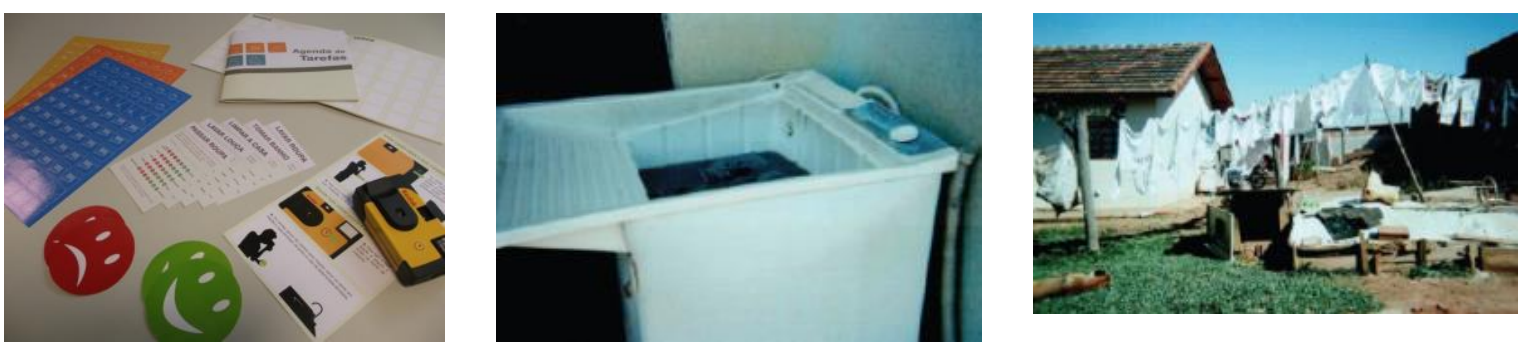

Figura 1 - Kit de sondas culturais (à esquerda), tanquinho utilizado para lavar as roupas (ao centro), Roupas penduradas no varal (à direita). Fonte: (Os Autores).

Posteriormente, foram realizadas as entrevistas semiestruturadas com as 7 famílias (Daros, 2013). Nessa etapa foram aprofundados diversos aspectos sobre os hábitos de higiene, as atividades domésticas, o consumo de água, energia e produtos de limpeza e higiene, percepções, tecnologias, informação, dentre outros. A partir das sondas culturais e das entrevistas, foram desenvolvidos esquemas visuais representando os procedimentos adotados nas atividades domésticas e de higiene. Desse modo, foi possível constatar as semelhanças e as divergências entre as famílias. Para auxiliar no processo de análise, os dados coletados pelas entrevistas foram transcritos e codificados destacando aspectos como as atitudes, os interesses e opiniões dos entrevistados (Vyncke, 2002). Parte dos diálogos foi separado para reforçar as impressões durante as práticas para construção dos cenários de futuro por grupos multidisciplinares de especialistas.

A análise das sondas possibilitou entender que as práticas que formam o hábito de consumo de água e energia estão relacionadas aos aspectos culturais (ensinamentos adquiridos formalmente e pela família, o papel da mulher e da dona de casa, e do resultado direto do processo de limpeza), ao contexto (habitação e comunidade), à rotina doméstica, aos artefatos utilizados e à concepção de limpeza e higiene. Ressalta-se, principalmente, os conceitos de limpeza e a higiene, pois estes estão diretamente relacionados com o consumo de água e energia. Além disso, todos esses fatores apontam possibilidades de inovação a serem explorados em produtos e serviços (Daros, 2013). 
Um banco de dados com inovações foi montado considerando informações sobre o tipo de produto ou serviço, sua descrição detalhada, as tecnologias aplicadas em seu funcionamento, as estratégias adotadas para reduzir o impacto ambiental, o país de origem e algumas palavras-chaves para agrupar as soluções. Após o levantamento, foi possível reunir um número expressivo de soluções e tecnologias considerando desde os primeiros produtos na história até as propostas conceituais de futuro ou o uso de tecnologias alternativas, como novos processos químicos, mecânicos ou elétricos.

A partir dos dados analisados, workshops colaborativos e multidisciplinares foram promovidos para a criação de cenários de futuro. Essas práticas contaram com a participação de arquitetos, assistentes sociais, engenheiros químicos, civis e eletricistas, designers, representantes da indústria da linha branca e representantes da COHAPAR. Para a sua realização, os workshops tomaram como referência relatórios globais desenvolvidos pelo International Food Policy Research Institute (Rosegrant, Cai, \& Cline, 2002), pelo World Energy Council (2007) e pela UNESCO (Gallopín, 2012). Os macro cenários criados foram agrupados em visões de futuro que retratassem uma "crise hídrica e energética", um "consumo sustentável de água e energia" e uma visão intermediária de "transição do consumo de água e energia". As informações selecionadas para a contextualização e descrição dos cenários correspondiam a sociedade, a tecnologia, a economia, a ecologia e as políticas, conforme Augsten (2011).

Como resultado dessa etapa, o cenário de "crise hídrica e energética" nas HIS foi caracterizado pelo fomento de soluções criativas, sendo este cenário o mais propício para as inovações e o empreendedorismo. Pela escassez apontada no cenário, seriam desenvolvidos produtos e serviços que realizariam as tarefas domésticas e de higiene com o mínimo de consumo de água e energia, e o desperdício seria praticamente eliminado. Nesse cenário, o consumo seria contido. A "transição do consumo de água e energia" considerava a continuidade dos hábitos atuais oferecendo melhorias incrementais relacionadas a ecoeficiência. E, no cenário de "consumo sustentável", haveria o incentivo ao desenvolvimento de novos produtos e serviços, bem como, a adoção de hábitos racionais de consumo de água e energia. Esse cenário seria viável em função da educação ambiental e das tecnologias sustentáveis aplicadas aos produtos e serviços.

A construção dos cenários e as discussões contribuíram para a definição de resultados mais elaborados. A participação de especialistas foi considerada fundamental para a 
reelaboração consolidada sobre o entendimento dos conceitos de limpeza, as proposições de soluções focadas em produtos sustentáveis partindo do uso de tecnologias, dos espaços compartilhados e de convivência social. Todos esses aspectos foram considerados importantes para a concepção de um novo sistema econômico e de políticas públicas para promover novos padrões de consumo e mudanças nas cadeias produtivas.

As diretrizes metaprojetuais buscaram inovar instrumentalizando o desenvolvimento de novos produtos e serviços. Essas diretrizes atenderam um ou mais critérios ou estratégias relacionadas ao consumo de água e energia, hábitos de consumo, sustentabilidade e inovação. No caso do presente estudo de caso, as diretrizes metaprojetuais contemplaram proposições relacionadas ao abastecimento de água e no fornecimento de energia para as HIS e a comunidade, os artefatos envolvidos nas atividades de lavar roupas, o hábito de consumo de água e energia nas atividades investigadas e no uso da lavanderia.

\section{DISCUSSÕES}

A partir da aplicação do marco preliminar num estudo de caso, foi possível analisar os instrumentos, a aplicação das ferramentas, verificar a qualidade e pertinência dos resultados obtidos e, por fim, identificar as melhorias nos instrumentos e procedimentos. Para cada etapa, os instrumentos de coleta de dados foram testados em atividades piloto considerando a coleta, o tratamento e a análise dos dados. Esse procedimento permitiu corrigir falhas, evitar coletas de dados redundantes e ampliar informações relevantes à pesquisa. Destacase que os processos adotados para a elaboração e organização seguiram esse padrão para as questões e estrutura da survey, da seleção e elaboração dos materiais que compuseram os kits das sondas culturais, as perguntas e estrutura da entrevista semiestruturada, e as orientações das atividades dos workshops para construção dos cenários futuros. A organização consistiu em priorizar aspectos e reunir aqueles que destacassem as experiências, as percepções, as opiniões e as necessidades dos consumidores sobre cada atividade observada. Para cada etapa do marco teórico foi estabelecido um protocolo detalhado sobre as ações necessárias para a coleta de dados, o tratamento dos dados e a estratégia adotada para as análises.

Após a observação dos procedimentos e resultados, foram identificadas 3 melhorias. A primeira refere-se ao mapeamento das atividades domésticas nas fases preliminares da pesquisa. Entende-se que esse mapeamento poderia ajudar na identificação de critérios 
objetivos de consumo, permitindo comparar as percepções das famílias sobre seu consumo e os dados de consumo nacionais e internacionais publicados em órgãos oficiais. Caso o mapeamento seja realizado em fases posteriores, considerando a percepção dos sujeitos investigados, o componente subjetivo pode modificar os aspectos considerados importantes para descrever o real impacto sobre o consumo na renda familiar. A segunda sugestão diz respeito ao banco de inovações que poderia incluir além dos critérios ambientais, contribuições sociais, culturais, econômicas, questões legais e normativas que se relacionam com as políticas públicas. A inclusão desses aspectos poderia conferir maior equilíbrio na análise e discussão das propostas atendendo aos três pilares da sustentabilidade. Além disso, inserir informações relacionadas a prospecção das tecnologias, orientada por métodos inventivos como o TRIZ (Lovel, Seastrunk, \& Clapp, 2006; Savransky, 2005) e orientada para a criação de cenários futuros. Nesse contexto, considera-se importante a automação da trajetória do produto e serviço para o banco de inovações.

A terceira sugestão poderia ampliar o desenvolvimento de sistemas de produtos e serviços sustentáveis por meio de workshops. A intenção seria aprofundar as discussões sobre a viabilidade e implementação como alternativa às demandas de futuro da comunidade. Essa melhoria ajudaria a ampliar o leque de soluções e criar novos modelos de negócios para atender a comunidade observada. Com a implantação dessas melhorias, acredita-se que seja possível aprimorar o marco preliminar, visando o estabelecimento de um modelo teórico orientado para designer e contribuindo para a elaboração de diretrizes metaprojetuais sustentáveis.

Constata-se que, para a aplicação do marco preliminar num estudo de caso, a estrutura composta por duas partes e sete atividades possibilitou o atendimento das expectativas iniciais do projeto de propor uma agenda de inovação a partir da identificação de oportunidades de inovação, considerando os hábitos de consumo. Essas partes foram caracterizadas como descritivas. Nesse sentido, explicita-se que a etapa de coleta e análise dos dados foi composta pela definição do público, a aplicação de uma survey, das sondas culturais e de entrevistas semiestruturadas para identificar os hábitos de consumo de água e energia no contexto atual. A segunda etapa do marco correspondeu a elaboração de um banco de inovações, dos cenários de futuros e das diretrizes metaprojetuais orientadas para mudar o comportamento sustentável por meio dos resultados da primeira etapa. O estudo de caso contribuiu para entender as particularidades e contribuições dos métodos mistos de 
pesquisa. Cada instrumento adotado forneceu contribuições importantes para a pesquisa e os pesquisadores participantes do grupo.

\section{CONCLUSÕES}

O presente trabalho apresentou a experiência adquirida na aplicação de um marco preliminar de pesquisa em design para elaborar uma agenda de inovação. O marco preliminar que combinou métodos qualitativos, foi dividido em compreender os hábitos de consumo no contexto atual, construir cenários de futuro e elaborar diretrizes para promover mudanças de comportamento de consumo, inovação e sustentabilidade. Através da aplicação do marco foi possível confirmar a hipótese de que é possível estabelecer um conjunto de diretrizes amplas que possam orientar os atores de projeto tomando como referência o conhecimento sobre os hábitos atuais e os contextos em que os usuários estão inseridos. A aplicação do marco preliminar proporcionou verificar pontos de melhoria na estrutura, dos instrumentos, os protocolos de coleta de dados, os protocolos de tratamento de dados e estratégias de análise.

Como contribuição, o presente estudo mostrou que é possível conhecer os hábitos de consumo e, a partir desse conhecimento, propor diretrizes metaprojetuais para orientar o setor público na elaboração de políticas públicas e os designers nos processos de desenvolvimento de novos produtos e serviços. A aplicação contou com a participação de equipes multidisciplinares e especialistas para construir instrumentos de coleta de dados, realizar workshops e analisar dados. Além disso, destaca-se a participação dos consumidores e usuários como atores ativos no processo de pesquisa favorecendo sua contribuição e permitindo expressar suas experiências, opiniões, expectativas e necessidades durante a primeira parte da investigação. $O$ trabalho ajudou a reconhecer as potencialidades sobre o uso dos métodos mistos no campo do design. Para a continuidade do presente estudo, três propostas são apresentadas:

(a) Considerar a participação de outros instrumentos para compreender o comportamento de compra do consumidor que seria importante para explorar as razões que levam a tomada de decisões.

(b) Aplicar o marco preliminar em outros contextos de hábitos de consumo e perfil do público, utilizando instrumentos como o videotour e o shadowing, a fim de identificar falhas no uso dos produtos, problemas não percebidos de forma consciente pelos consumidores em certas tarefas domésticas, as falhas de interpretação durante o uso dos produtos e as adaptações 
implementadas para aperfeiçoar as funções dos produtos. Nesse contexto, poderia ser estudada a integração de tecnologias digitais conectadas ou o uso de dispositivos móveis para acompanhar e registrar em tempo real as rotinas e permitir outras interações com o público observado.

(c) E, propor um modelo que permita identificar e avaliar mudanças nos hábitos de consumo a partir das soluções que foram desenvolvidas com base nas diretrizes metaprojetuais ou das mudanças causadas pelas políticas públicas. Essa avaliação poderia ajudar a identificar quais aspectos foram afetados nas rotinas, quais os impactos na economia familiar e nas atividades do cotidiano dos consumidores, quais as diretrizes que precisam ser revisadas e quais aspectos não foram contemplados na agenda de inovação.

\section{REFERÊNCIAS}

Augsten, A., Böttger, M., Carsten, S., \& Engel, L. (2011). Urban Futures 2050 - Szenarium - Szenarien für die Zukunft der Städte Europas. Berlin, Germany.

Barr, S., Gilg, A., \& Shaw, G. (2011). 'Helping People Make Better Choices': Exploring the behaviour change agenda for environmental sustainability. Applied Geography, 31(2), 712-720.

Bocken, N. M. P., Mugge, R., Bom, C. A., \& Lemstra, H.-J. (2018). Pay-per-use business models as a driver for sustainable consumption: Evidence from the case of HOMIE. Journal of Cleaner Production, 198, 498-510.

Celaschi, F., \& Deserti, A. (2007). Design e innovazione: strumenti e pratiche per la ricerca applicata. Carocci Roma.

Chu, W., Glad, W., \& Wever, R. (2019). Embracing Change While Retaining the Existing: Sustainable Behaviour Design Insights from Astronaut Food Consumption Transitions. IASDR Conference 2019.

Chu, W., Steenstra, P., Wever, R., \& Glad, W. (2018). Understanding context change: An activity theoretical analysis of exchange students' food consumption. In Proceedings of NordDesign: Design in the Era of Digitalization, NordDesign 2018.

Daros, C. (2013). Design para a sustentabilidade: oportunidades de inovação a partir dos hábitos de consumo na habitação de interesse social. 2013. 183 f(Dissertação de mestrado). Programa de Pósgraduação em Design - PPGDesign/UFPR, Curitiba, Paraná.

Daros, C., Camelo, D. M., Silvério, D., \& Santos, A. dos. (2014). Avaliação qualitativa de hábitos de consumo: resultados preliminares a partir do método das Sondas Culturais. In Anais do 40 Encontro de Coordenadores da Rede de Pesquisa sobre o uso racional de água e eficiência energética em Habitações de Interesse Social (pp. 35-76). São Cristóvão, Sergipe, Brasil: Editora UFS.

Elizondo, G. M., \& Lofthouse, V. (2011). Patterns of conservation and domestic water use in different cultures: a comparison between Mexico and the UK.

Gallopín, G. C. (2012). Five stylized scenarios. Global Water Futures, 2050.

Godet, M. (2001). Creating futures: scenario planning as a strategic management tool. Paris, France: Economica.

Lilley, D. (2007). Designing for behavioural change: reducing the social impacts of product use through design (PhD Thesis).

Lilley, D. (2009). Design for sustainable behaviour: strategies and perceptions. Design Studies, 30(6), 704-720. 
Lockton, D., Harrison, D., \& Stanton, N. A. (2010). The Design with Intent Method: A design tool for influencing user behaviour. Applied Ergonomics, 41(3), 382-392.

Lockton, D., Harrison, D., \& Stanton, N. A. (2016). Design for Sustainable Behaviour: investigating design methods for influencing user behaviour. Annual Review of Policy Design, 4(1), 1-10.

Lovel, K., Seastrunk, C., \& Clapp, T. (2006). The application of TRIZ to technology forecasting a case study: Brassiere strap technology. TRIZ Journal, www. Triz-Journal. Com.

Lucero, A., Lashina, T., Diederiks, E., \& Mattelmäki, T. (2007). How probes inform and influence the design process. In Proceedings of the 2007 conference on Designing pleasurable products and interfaces - DPPI '07 (p. 377). Helsinki, Finland: ACM Press.

Marinova, D. (2009). Global Green System of Innovation: Technological Wave or Policy? In Proceedings of MODSIM 2009 International Congress on Modelling and Simulation (pp. 1168-1174). The Modelling and Simulation Society of Australia and New Zealand.

Porter, A. L., Ashton, W. B., Cuenter, C., Joseph, F. C., Kerstin, C., Scott, W. C., \& Ken, D. (2004). Technology futures analysis: Toward integration of the field and new methods. Technological Forecasting and Social Change, 71(3), 287-303.

Rosegrant, M. W., Cai, X., \& Cline, S. A. (2002). World water and food to 2025: dealing with scarcity. Washington, D.C: International Food Policy Research Institute.

Santos, A. dos, \& Daros, C. (2014). O hábito de lavar roupas (1a ed.). Editora Insight.

Savransky, S. D. (2005). Engineering of creativity: introduction to TRIZ methodology of inventive problem solving. Boca Raton, Fla: CRC Press.

Tamborrini, P. (2014). Innovation Design. In A. C. Michalos (Ed.), Encyclopedia of Quality of Life and Well-Being Research (pp. 3272-3278). Dordrecht: Springer Netherlands.

Tang, T., \& Bhamra, T. (2008). Changing energy consumption behaviour through sustainable product design. In DS 48: Proceedings DESIGN 2008, the 10th International Design Conference, Dubrovnik, Croatia.

Vezzoli, C., \& Manzini, E. (2008). Design for environmental sustainability. [Berlin] ; London: Springer.

Vyncke, P. (2002). Lifestyle Segmentation: From Attitudes, Interests and Opinions, to Values, Aesthetic Styles, Life Visions and Media Preferences. European Journal of Communication, 17(4), 445-463.

World Energy Council. (2007). Deciding the future energy policy scenarios to 2050. London: World Energy Council. 